\title{
Moments (inter)discursifs et emplois métaphoriques du champ dérivationnel de "ciment" dans la presse Chypriote-grecque. Une approche pragmatico-sémantique
}

\author{
Maria Constantinou (Philips College, Nicosie)
}

\begin{abstract}
This article intends to closely investigate a particular ad hoc metaphor, which concerns the

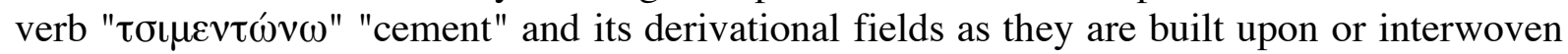
with dynamic intertextual networks in a newspaper. The diversity of usages that this metaphor goes through raises the problem of the semantic displacement or innovation. The study of the metaphorical use of the verb "cement" and its derivational field in a variety of contexts where they appear, leads one to problematize it in the light of the 'trigger utterance', historically and ideologically charged. In other words, the metaphor should be investigated within discursive and intertextual relations, which seem to be established in a newspaper. Semantic movements, multiple interpretations and readings, ambiguity and argumentation are the main problems that this metaphor typically raises in the present study. A textual, enunciative and componential approach will be here adopted.
\end{abstract}

\section{Introduction et contexte historique}

Dans cet article nous nous proposons d'explorer, dans une perspective pragmatico-sémantique l'usage métaphorique du verbe $\tau \sigma \mu \mu \varepsilon v \tau \omega \nu \omega$ "cimenter" et de son champ dérivationnel $\tau \sigma \iota \mu \varepsilon ́ v \tau \omega \mu \alpha$ "cimentation", $\alpha \pi O-\tau \sigma \iota \mu \varepsilon v \tau \omega \dot{v} \omega$ "dé-cimenter" etc., tels qu'ils se voient utilisés dans le discours de la presse écrite chypriote grecque. Le choix de cette métaphore particulière comme objet d'étude d'une part se justifie par l'usage qu'en ont fait les journalistes dans un contexte bien déterminé de la vie nationale à Chypre, et d'autre part par la prolifération incontrôlée de sens que cimenter et son champ dérivationnel suscitent dans le discours médiatique.

Notre étude a pour objet la diversité des usages métaphoriques du ciment tels qu'ils se déploient au sein d'un même quotidien, après un énoncé métaphorique produit par $\mathrm{D}$. Christofias $^{1}$, à l'époque chef du Parti progressiste des travailleurs (AKEL) et président de la Chambre des Représentants. Cet énoncé semble avoir déclenché chez les professionnels de la

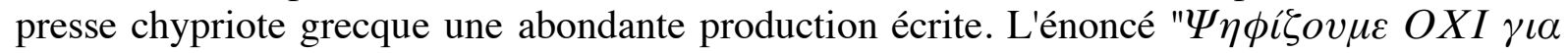

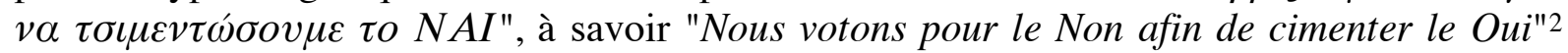
s'est produit à une période critique pour l'histoire récente de la République de Chypre. En effet, il s'agit du référendum pour le Plan Annan qui s'est déroulé à Chypre le 24 avril 2004 en vue de la réunification de l'île.

\footnotetext{
${ }^{1}$ D. Christofias a été élu président de la République de Chypre le 24 février 2008.

${ }^{2}$ Le positionnement de cet homme politique a beaucoup pesé sur le verdict du référendum.
} 
A l'exception de certains de ses membres qui se sont initialement posés en faveur du plan onusien, le président de l'ex- Parti communiste évite de prendre une position nette. L'AKEL ${ }^{3}$ et le parti d'opposition DISY dont les orientations penchaient vers le "Oui", décident de reporter leur décision à des congrès organisés peu avant le référendum. C'est à un peuple abreuvé des arguments contre le plan Annan, que T. Papadopoulos, à l'époque président de la République de Chypre, s'adresse, dans la finalité de plaider contre le "Oui". Face à ce climat tendu et irréversible en faveur du "Non", qui divise la communauté chypriote grecque en deux camps, et par peur de perdre ses électeurs, la majorité du comité central de l'AKEL renonce à soutenir le plan onusien. Christofias, pour parer au danger d'un schisme de son parti, sollicite le report du référendum, et deux jours avant le scrutin, se voit finalement obligé d'opter pour un Non "tiède", "doux".

\subsection{Corpus}

Faute d'avoir accès aux archives des médias de masse télévisuels, nous nous sommes limités aux archives électroniques de la presse écrite de la période allant de l'année 2004 (fin avril) à 2006 qui sont accessibles. Le corpus est constitué des articles tirés exclusivement du quotidien chypriote grec Simerini. Pour nous limiter au discours journalistique proprement dit, nous avons exclu de notre corpus de base la rubrique "opinions". Nous avons également exclu les quotidiens chypriotes grecs Phileleftheros et Politis qui n'étaient pas accessibles. Nous avons recensé 143 articles dans lesquels apparaissent soit le verbe "cimenter" soit ses dérivés, dont 56 articles en 2004, 47 en 2005 et 40 en 2006. Dans ces articles nous avons inventorié 210 occurrences. Le verbe "cimenter" dans ses sens métaphoriques apparaît 159

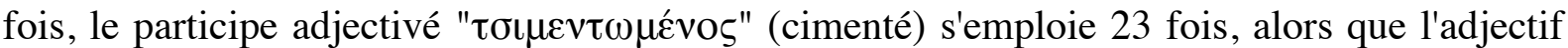

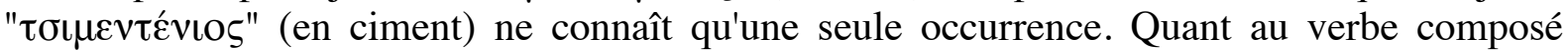

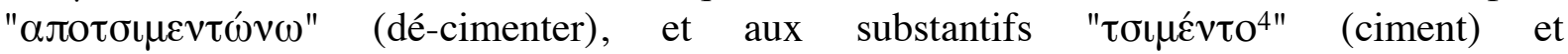

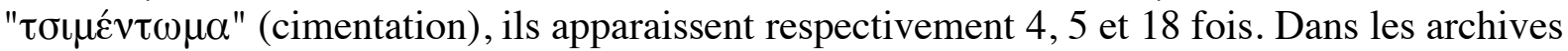
électroniques le signifiant se déploie sous sa forme métaphorique en 2002 et en 2003, mais avec une fréquence très faible ( 3 occurrences pour 2002 et 6 pour 2003).

La lecture du quotidien Simerini nous a permis de constater que les journalistes-scripteurs, recourent au verbe cimenter et à son champ dérivationnel pour marquer leur opposition ou désapprobation envers des événements qui se déroulent au sein de la communauté chypriote grecque. Alors que les occurrences chargées de traits négatifs remontent à 208, celles qui actualisent des traits positifs ne dépassent guère les 5 , ce qui nous permet de postuler que l'emploi métaphorique du 'ciment' renvoie inévitablement à cette expérience relative au contexte historique, profondément ancré dans la mémoire collective de la communauté chypriote grecque. Il est également opportun d'observer que les traits positifs ne s'actualisent que lorsque les journalistes se réfèrent soit à la volonté du peuple $(1 \text { occurrence })^{5}$, soit au

\footnotetext{
3 Notons que l'AKEL a toujours prôné les négociations et les filiations amicales entre les deux communautés, (chypriote grecque et chypriote turque) en vue de la réunification de l'île. C'est le seul parti politique qui ait parlé d'une identité pouvant réunir les deux communautés, celle du chypriotisme.

${ }^{4}$ Le substantif "ciment" apparaît dans son sens métaphorique. Chargé de traits négatifs, il signifie "fondement", "socle". Voici un exemple : Le premier ciment de la division s'est jeté après la création de l'enclave-ghetto entre les quartiers chypriotes grecs de Nicosie et de Kyrenia. Dès lors, milliers de tonnes en ciment se sont jetés à tous les égards de l'affaire chypriote, pour finir à l'heure actuelle par entendre parler de division par le

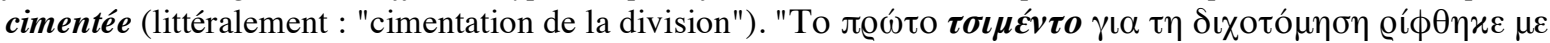

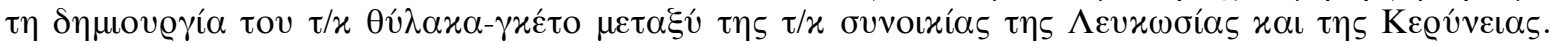

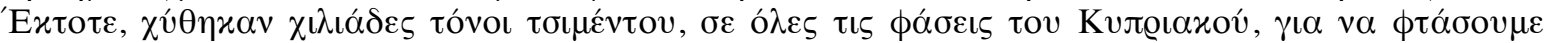

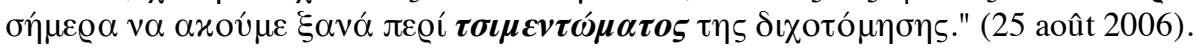

${ }^{5}$ Qu'est-ce que les Etats-Unis réussissent à faire? Premièrement, ils suscitent la fureur, et animent le sentiment anti-américain des Chypriotes. Deuxièmement, ils cimentent la volonté du peuple chypriote à résister encore

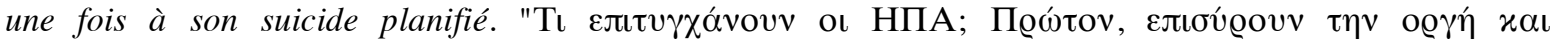


président Tassos Papadopoulos (3 occurrences) ${ }^{6}$. Une autre occurrence qui actualise des traits positifs sera analysée dans cette étude (ex. 14).

La prise en compte du nombre d'occurrences et les avatars sémantiques de cette métaphore nous amènent à ne présenter que des exemples tirés de la titraille ${ }^{7}$. Ce choix se justifie par le fait que la titraille, notamment le titre et le sous-titre constituent la composante la plus importante de l'article. D'ailleurs, la titraille est censée révéler au lecteur le contenu de l'article, sans qu'il soit nécessaire de lire tout le texte (cf. Furet 1995) ${ }^{8}$. Au sein du journal Simerini, les titrailles comportant la métaphore du ciment, semblent afficher davantage le point de vue ou la posture auctoriale du journaliste. La métaphore du ciment, renforcée par la mise en valeur éditoriale de la titraille redouble dans son contenu suggestif. Basée sur une expérience commune, elle semble renforcer la fonction 'phatique' des titres et sous-titres qui fonctionnent comme 'incitateurs' à la lecture des articles. Les articles recensés qui incluent dans leurs titrailles la métaphore du ciment sont au nombre de 28 dont 7 ne portent pas directement sur le contexte purement politique. Nous avons recensé 19 titres, 5 sous-titres, 2 chapeaux et 2 intertitres. Dans la plupart des articles il y a reprise de la métaphore dans le contenu des articles (16/28), ce qui contribue à établir des isotopies intra- et intertextuelles. Les récurrences vont de 1 à 6 ( 1 occurrence : 10 articles, alors que pour les articles l'analogie se répartit de la manière suivante $: 2: 2,3: 2,4: 1,5: 0,6: 1)$.

\subsection{Problématiques du travail et hypothèses}

La prise en compte du contexte et de la situation historique, ainsi que le déplacement du "mapping", à savoir l'identification métaphorique, semblent compliquer le cadre théorique dans lequel nous cherchons à inscrire notre étude. Nous nous interrogeons, dans un premier temps, sur le cadre théorique du point de vue de la métaphore, autrement dit, nous cherchons à savoir quelles sont les règles qui régissent le processus métaphorique de cette métaphore où le signifiant qui fonctionne comme "oscillateur sémantique"9 actualise des significations diverses.

Dans une perspective sémantique, nous étudions cette métaphore du point de vue de la polysémie et de l'ambiguïté. Il s'agit de nous interroger sur l'appréhension de ces significations hétérogènes, à l'évidence, liées au sens que le mot a initialement pris dans cet énoncé historiquement et idéologiquement déterminé et déployé en réseaux intertextuels dynamiques. Le contexte dans lequel cette métaphore apparaît ainsi que les jeux de mots ou tropes qui l'accompagnent ou que celle-ci entraîne méritent également notre attention.

Dans une optique pragmatique, il convient de nous interroger sur les valeurs argumentative et évaluative de la métaphore. Nous nous posons la question de savoir comment l'usage de la métaphore du ciment contribue-t-il à la construction argumentative et évaluative des articles.

Ces considérations nous amènent à avancer les hypothèses suivantes :

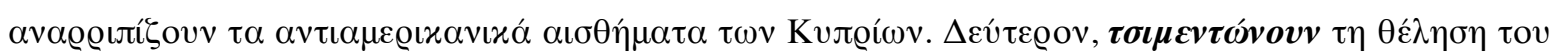

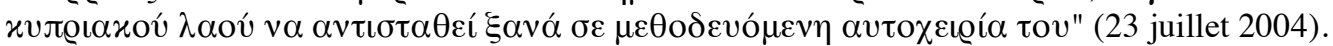

${ }^{6}$ Tassos Papadopoulos est incontestablement le vainqueur du lendemain. Les perspectives pour le président de se faire réélire se cimentent grâce à l'approbation de la ligne politique qu'il suit à l'égard de l'affaire

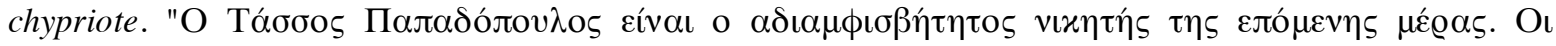

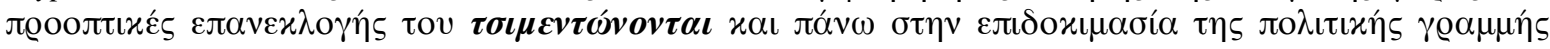

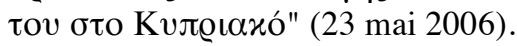

7 Cependant, pour des fins statistiques, tout au long de notre analyse nous aurons recours à l'ensemble des exemples inventoriés.

${ }^{8}$ Du point de vue du lecteur, Furet note que la titraille : "doit être comprise sans qu'il soit nécessaire de lire le texte" (1995: 104).

${ }^{9}$ Le terme est emprunté à Jean-Pierre Faye. 
a) l'instabilité du sens de cette métaphore (ceci est dû à la polysémie et l'ambiguïté que celle-ci comporte) favorise des emplois qui donnent naissance à des significations hétérogènes.

b) grâce à la prolifération de sens "incontrôlés", les journalistes se servent de cette métaphore soit pour combler un vide linguistique, soit pour créer une "réalité" politique ou sociale qui n'est pas dénuée de connotations moqueuses. Cela revient à admettre qu'une interprétation adéquate pour chaque cas particulier ne se fait que grâce à la complicité du contexte et de nos connaissances du monde.

c) les journalistes en se servant de la métaphore créent une polémique et énoncent leurs positions sur différents événements. Dans cette perspective, la métaphore se voit comme un outil à afficher les convictions politiques du journal et elle témoigne d'une volonté de persuader le lecteur de la justesse de celles-ci.

\section{Balisage théorique}

Notre propos appelle par définition un cadre théorique pouvant intégrer la problématique de la métaphore, la notion d'argumentation, celle de l'intertextualité, et les relations sémantiques complexes que la métaphore du "ciment" tisse dans une multitude de contextes où celle-ci apparaît. Dans un premier temps, nous essayerons de cerner la métaphore d'abord dans sa fonction cognitive pour ensuite étudier ses fonctions argumentative et évaluative. Dans un second temps, nous interrogerons cette métaphore sous l'angle de l'intertextualité et des parcours interprétatifs que celle-ci entraîne.

\subsection{La métaphore : quelques réflexions théoriques}

\subsubsection{Métaphore ad hoc}

A la suite de Ricoeur (1975) les linguistes ont tendance à établir la différence entre métaphore morte et métaphore vive. Le premier type déjà lexicalisé relève du cliché alors que le deuxième tient de l'invention. La métaphore vive sert à désigner une métaphore originale et neuve, qui s'oppose à la métaphore morte. Par métaphore morte ou lexicalisée on entend cette figure rhétorique dont le sens est reconnu et répertorié dans le dictionnaire et utilisé couramment. "La racine du mal", ou "être sur la sellette" font partie de ce type de métaphore.

Or, cette dichotomie pose problème, et nous nous demandons avec R. Landheer (2002: 29) s'il s'agit d'une répartition pertinente..$^{10}$ L'auteur avance deux causes qui remettent en question la pertinence de cette dichotomie. La première tient à la différence de compétence linguistique des locuteurs ; "une métaphore morte, écrit-il, pour l'un pourrait bien être une métaphore originale, vivante pour quelqu'un d'autre." La deuxième cause "réside dans le fait qu'un sens métaphorique a beau être usé, éteint, il peut à tout instant être ranimé discursivement." (ibid.). Autrement dit, chaque acte d'énonciation dans sa singularité est susceptible de redynamiser et donc "recycler" le sens métaphorique d'un terme ou d'une expression.

I. Collombat (2005 : 36) récuse le terme de métaphore vive, puisqu'il "évoque [...] trop systématiquement la métaphore 'décorative'"11 et préfère parler de métaphore ad hoc, car il "marque davantage le caractère exprès du trope lorsqu'il est original, créé par un usage

\footnotetext{
${ }^{10}$ L'auteur démontre que le terme "métaphore morte" est mal choisi, car "un sens métaphorique contient toujours un germe de vivacité, une sorte de fluidité insaisissable, et par là même une espèce d'ambiguïté fondamentale." (ibid. : 29).

${ }^{11}$ Dans notre perspective, la métaphore n'est pas considérée comme un simple trope. Elle ne s'envisage non plus sous le prisme du Groupe $\mathrm{M}$ qui pour expliquer la métaphore en tant que trope, fait appel à la notion d'écart par rapport à un degré zéro.
} 
du champ dérivationnel de "ciment" dans la presse Chypriote-grecque.

unique dans un contexte donné, doté d'une visée propre, non lexicalisé et peu ou pas entré dans l'usage." Dans cette perspective, le terme proposé par l'auteure répond mieux, nous semble-t-il, à la problématique de la présente étude.

\subsubsection{Du point de vue cognitif}

Le vocable ciment selon la définition donnée par Le Petit Robert ${ }^{12}$ sert à désigner une "matière pulvérulente, à base de silicate et d'aluminate de chaux, obtenue par cuisson et qui, mélangée avec un liquide, forme une pâte liante, durcissant à l'air ou dans l'eau 13". Dans cette optique, la métaphore ad hoc utilisée par Christofias en fonction d'un contexte discursif particulier, est à l'évidence fondée sur ce que Lakoff et Johnson nomment les "espèces naturelles d'expérience"14. Elles sont en effet issues à partir de nos interactions avec l'environnement physique ou avec d'autres individus au sein de notre culture (cf. Collombat, 2005). Cette thèse rappelle le postulat de Swadesh (1955) selon lequel les concepts humains universaux sont éventuellement déterminés par les conditions universelles de la vie humaine, principalement de l'environnement dans lequel évolue l'être humain ; il s'agit en effet des éléments physiques tels que le soleil, les étoiles, la lune, la pluie, l'eau, le vent etc.

Sous cet angle, la métaphore peut se définir comme une mise en relation identificatrice, comme une série de correspondances conceptuelles s'opérant entre deux domaines; l'un est le domaine source et l'autre le domaine cible. Dans la terminologie anglo-saxonne, ce phénomène est connu sous le terme de "mapping" (cf. Jamet 2003).

Or, la prise en compte des avatars du sens métaphorique du verbe "cimenter" et de ces dérivés ne permet pas d'adopter à la lettre le modèle cognitif de l'identification métaphorique. Précisément, si le champ conceptuel de la métaphore "Le ciment est du pouvoir" ou "cimenter c'est renforcer" répond parfaitement à l'énoncé "déclencheur ${ }^{15 ",}$ il s'avère pour autant peu

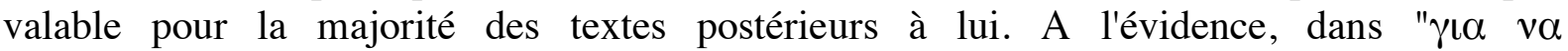

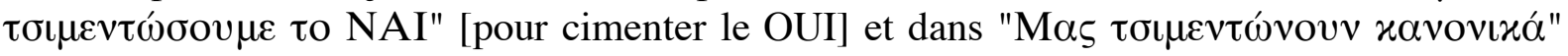
[Ils nous font cimenter] ${ }^{16}$, le signifiant en question n'actualise ni le même sens ni les mêmes sèmes (éléments de signification) et par là même n'amène guère aux mêmes parcours interprétatifs.

\subsection{Fluctuation sémantique, interprétation et contexte}

Kocourek (1991) qui souligne "la force et la faiblesse de la motivation métaphorique", indique, d'un côté, qu' "il y a la valeur picturale, le pouvoir d'évocation, le caractère concret et vif", d'un autre côté, qu' "on constate le manque de systémicité, l'absence d'indications

\footnotetext{
12 Notons que contrairement à la langue française où l'emploi métaphorique des mots ciment et cimenter est lexicalisé, c'est-à-dire il est rentré dans le dictionnaire, le recours à la lexicographie grecque (Babiniotis 2002) nous révèle qu'il n'y a pas eu jusqu'à lors de fossilisation de cette métaphore. Un examen sur le Net nous a révélé qu'un tel emploi métaphorique du verbe en question ne se fait que par la presse chypriote grecque et non pas par la presse grecque.

${ }^{13}$ Ciment et $\tau \sigma \iota \mu \varepsilon ́ v \tau o$ dans leur fonction référentielle s'avèrent identiques. Cependant, du point de vue métaphorique , le nom "ciment" et le verbe "cimenter" comme l'atteste le même dictionnaire est synonyme de "raffermir", "lier", "consolider" "unir", "sceller" etc.

14 Traduction de Michel Defornel $1985: 127$, cité par Collombat $2005: 222$.

15 Nous avons introduit, faute de mieux, l'expression "énoncé déclencheur" pour désigner l'énonciation qui a déclenché une abondante production écrite et orale chez les professionnels de la presse et les lecteurs. Nous avions également pensé au terme "énonciation d'origine", cependant, les premières occurrences du ciment dans son emploi métaphorique, qui remontent chronologiquement avant les propos de D. Christofias, nous interdisent un tel choix.

16 Voir l'exemple 9 de cet article.
} 
objectives et, en particulier, l'ambiguïté, c'est-à-dire l'applicabilité de la même métaphore à une quantité trop élevée de cas différents". (1991 : 170, gras de l'auteur).

Il s'agit en effet d'une sorte de conflit (Beardsley 1962 : 298) de sens, ou mieux encore d'une fluctuation sémantique issue des contextes divers dans lesquels cette métaphore apparaît. Les déplacements sémantiques s'effectuent par activation ou inhibition sémiques (par exemple les traits /force/, /mélioratif/ etc. qui se voient contredits dans certains contextes) par rapport à l'énoncé déclencheur. Ainsi, le lecteur et notamment l'analyste se trouvent confrontés à des problèmes interprétatifs complexes. Il se pose alors la question de savoir quel sens conférer à chaque instance, et comment "cimenter" le sens qui semble être en constant mouvement.

La polysémie s'impose à l'analyse de l'actualisation du ciment et pose le problème de la régulation du sens. Le signifiant, fonctionnant comme "oscillateur sémantique" acquiert des propriétés supplémentaires qui entrent dans l'actualisation en interaction avec des éléments du contexte pour produire des effets de sens complexes. En conséquence, la métaphore en question semble se mouvoir dans un réseau sémantique très complexe qui ouvre inéluctablement la voie à l'ambiguïté et aux interprétations diverses et multiples qui y rivalisent.

Il est communément admis que la métaphore est censée saisir des idées non clairement définies dans et par l'expérience humaine. En d'autres termes, l'énonciateur qui a recours à une image opte pour un référent plus concret dans le but d'établir une relation de similitude avec une notion abstraite. Néanmoins, dans le cas de la métaphore du ciment, il n'en va pas ainsi. Sa mise en place dans ces énoncés opacifie et obscurcit son contenu. On peut alors avancer l'idée que le rapport ne réside pas que dans la ressemblance, entre "ciment" et "force" et ni entre abstrait et concret. Certes, l'énoncé déclencheur - dans lequel ces rapports sont valables - sert d'ancrage interprétatif, mais tout seul ne paraît pas adéquat pour l'interprétation du sens. Par conséquent, la métaphore du ciment est impérativement à saisir en fonction de son contexte d'emploi. ${ }^{17}$

Par ailleurs, comme le précise Horne (1992: 184) : "le terme métaphorique entretient non seulement un rapport paradigmatique, conceptuellement 'vertical', avec le terme qu'il est censé remplacer, mais également un rapport syntagmatique ('horizontal') avec les autres éléments de l'énoncé". De son côté, de façon analogue Murat (1981:327) souligne le caractère énonciatif de la métaphore; ainsi soutient-il que "la métaphore est un phénomène de discours ; sa spécificité rhétorique ne peut donc résulter que de la corrélation de facteurs syntaxiques, sémantiques et énonciatifs".

L'analyse sémique semble ici opératoire dans le sens où on cherche des éléments de sens permettant de mieux cerner les nuances et les déplacements sémantiques activés en contexte. Il nous revient donc de rappeler que le sème est un élément de signification ou un élément d'un sémème qui n'a pas d'existence sémantique en soi. Le sème est un trait différentiel qui sert à conjoindre ou disjoindre deux sémèmes et à établir entre eux une relation d'opposition ou d'équivalence (par exemple /animé/ et /inanimé/.) (cf. Rastier 1987, 1989).

Notons que le contenu épistémique que nous donnons aux notions d'"activation", et d'"inhibition" est différent de celui que propose Rastier, à qui nous empruntons ces termes. Ici le terme "inhibition" désigne l'interdiction des sèmes actualisés dans l'énoncé déclencheur et non en langue comme c'est le cas dans la Sémantique Interprétative, vu que la prolifération de l'usage métaphorique de ce terme a ses racines dans des énoncés antérieurs.

\footnotetext{
17 Nombreuses sont les études qui ont posé la primauté du contexte dans l'interprétation de la métaphore (Gibbs/Gerrig 1989, Inhoff, Lima/Carrol 1984 etc.).
} 
du champ dérivationnel de "ciment" dans la presse Chypriote-grecque.

La métaphore du ciment, comme tout discours médiatique se déploie dans un mouvement circulaire. C'est pourquoi son étude nous pousse un peu plus loin du contexte vers l'intertexte.

\subsection{1 (En)jeux intertextuels et métaphore comme expérience partagée}

Dans la perspective bakhtinienne cette métaphore reflète "le contexte et les contextes dans lesquels [elle] a vécu sa vie sociale intense..." (Bakhtine, cité pat Todorov 1981: 89). La métaphore du ciment n'est ni neutre ni exempte des énonciations qui la formulent ${ }^{18}$. Selon Kristeva qui a introduit ce terme "tout texte se construit comme mosaïque de citations, tout texte est absorption et transformation d'un autre texte" (1969: 85). Les travaux de cette auteure se situent dans la mouvance de Bakhtine, notamment par sa mise en oeuvre du concept d'intertextualité dont procède celui de "dialogisme". L'intérêt de ces études est qu'elles mettent en avant moins la notion de sources précises que celle d'inconscient du texte et de culture ambiante, cela sous l'angle de la "production" des textes et des oeuvres, de quelque nature qu'elles soient.

Dans le même ordre d'idée, l'intertextualité chez Barthes s'associe à des relations inéluctables, conscientes ou inconscientes entre différents textes. Précisément, écrit-il que :

Tout texte est un intertexte ; d'autres textes sont présents en lui, à des niveaux variables, sous des formes plus ou moins reconnaissables : les textes de la culture antérieure et ceux de la culture environnante ; tout texte est un tissu nouveau de citations révolues. [...] L'intertexte est un champ général de formules anonymes, dont l'origine est rarement repérable, de citations inconscientes ou automatiques, données sans guillemets. (Encyclopédia Universalis 1998 s.v. ("Texte (théorie du)")

Chez Riffaterre, l'intertextualité est à saisir comme effet de lecture, déterminée par le lecteur lui-même dans son action de lecture et de déchiffrement du texte. Selon l'auteur "l'intertextualité est la perception par le lecteur de rapports entre une ouvre et d'autres, qui l'ont précédée ou suivie" (Riffaterre 1980). Ainsi, les effets de sens produits par l'intertextualité sont tributaires de la capacité du lecteur à identifier dans les interstices du texte la présence de traces en provenance de textes antérieurs. Cette capacité change au fil du temps, selon la "mémoire" mise en jeu par les lecteurs à tel ou tel moment de l'histoire d'une culture.

Au sein de ce quotidien, l'intertextualité se présente comme un forum de débats, des conflits de paroles où les voix se lèvent pour dialoguer, et faire participer le lecteur soit avec le journaliste-scripteur, soit avec cette partie de la communauté partageant les mêmes opinions que le lecteur.

Sous cet angle, le jeu intertextuel que subit cette métaphore se met au service de la mémoire collective et se propose d' "unifier" une communauté. C'est pour cela que nous avançons l'idée que la dimension intertextuelle et interdiscursive ${ }^{19}$ de cette métaphore prend en compte au sein des jeux et enjeux du langage, les logiques et représentations de la communauté chypriote-grecque, avec ses repères historiques et idéologiques.

\footnotetext{
18 Comme le fait remarquer Bakhtine : "Aucun membre de la communauté verbale ne trouve jamais des mots de la langue qui soient neutres, exempts des aspirations et des évaluations d'autrui, inhabités par la voix d'autrui. Non, il reçoit le mot par la voix d'autrui, et ce mot en reste rempli. Il intervient dans son propre contexte à partir d'un autre contexte, pénétré des intentions d'autrui. Sa propre intention trouve un mot déjà habité" (cité par Todorov $1981: 77$ ).

${ }^{19}$ Notons que nous ne faisons pas ici une stricte distinction entre interdiscours et intertextualité puisque dans notre cas, les deux notions semblent s'enchaîner et se compléter mutuellement. Si l'interdiscours est la mise en relation réciproque et l'ouverture du discours sur d'autres discours, l'intertextualité est considérée ici comme la présence d'un texte dans d'autres textes.
} 


\subsubsection{Fonctions argumentative et évaluative de la métaphore du ciment}

J. B. Grize rappelle qu' "argumenter dans l'acception courante, c'est fournir des arguments, donc des raisons, à l'appui ou à l'encontre d'une thèse." Cependant, en plaidant pour une acception plus large du terme, il souligne la possibilité de concevoir l'argumentation "comme une démarche qui vise à intervenir sur l'opinion, l'attitude, voire le comportement de quelqu'un" (Grize, 1990: 41). Dans le cas de la métaphore du ciment, les journalistesscripteurs semblent y recourir pour convoquer les contextes dans lesquels elle est déjà apparue, avec la finalité d'apposer leur point de vue et/ou d'entraîner le lecteur dans telle ou telle direction.

Outre sa valeur argumentative, cette métaphore ad hoc semble se charger d'une autre fonction - qui révèle de l'argumentation même - celle de l'évaluation et de la dénonciation. Selon Bourdieu "les journalistes ont tendance à penser que le travail d'énonciation, de dévoilement des mécanismes, est un travail de dénonciation, dirigé contre des personnes, ou, comme on dit, des 'attaques' personnelles, ad hominem" (Bourdieu 1996 : 14-15).

En effet, le réemploi métaphorique et le recyclage sémantique de cette métaphore servent à renforcer une attitude de dénonciation et/ou de mise à distance critique par rapport aux événements et positions prises par tel ou tel homme politique. Dans cette optique, la métaphore laisse des marques énonciatives, car elle peut impliquer un jugement dévalorisant ou péjoratif. Vue sous cet angle, la métaphore du ciment n'est pas dotée que de vocation cognitive ou expressive ${ }^{20}$, autrement dit on est loin de l'idée qui présente la métaphore comme une simple enflure du discours.

Des auteurs ont mis en exergue le caractère évaluatif et énonciatif de la métaphore. $\mathrm{R}$. Kerzazi-Lasri (2003 : 106) par exemple souligne qu' "un énoncé métaphorique, en plus de son apport communicatif qui consiste à véhiculer des relations de détermination, de qualification, de comparaison, etc. assure d'autres fonctions implicites qui consistent à porter des jugements concernant les faits représentés."

Pour l'auteure, l'évaluation semble "dépendre de l'exercice inférentiel qui permet de comprendre la relation entre ce qu'indique un énoncé métaphorique et ce qu'il implique" (ibid. 107). De la sorte, les notions d'argumentation et d'évaluation ne se situent pas uniquement au niveau des mots mais elles sont tributaires de la pensée et des connaissances. L'évaluation relève donc de la compétence du lecteur qui peut ou non saisir les suggestions implicites d'un énoncé.

\section{$3 \quad$ Analyse du corpus}

La prise en compte de l'hétérogénéité sémantique, textuelle et intertextuelle de cette métaphore ad hoc oblige à faire une classification à la fois thématique et formelle. La classification se base d'abord sur une division thématique, entre énoncés se rapportant directement au plan onusien et à l'affaire chypriote, et énoncés liés à d'autres questions sociopolitiques. La multitude des contextes et des fonctionnements sémantiques que présente ce paradigme amène à procéder à une classification formelle.

\footnotetext{
${ }^{20}$ Précisons que la métaphore en question n'est dépourvue ni de fonction expressive ni de fonction cognitive. Comme nous le verrons par la suite l'expressivité est l'une des fonctions de cette métaphore. La vocation cognitive sert également d'ancrage interprétatif. C'est pour cela que nous adoptons volontiers le point de vue de Détrie (2001 : 250) qui refuse de faire une distinction entre métaphores poétiques, expressives, argumentatives et cognitives, vu que ce n'est qu'a posteriori que l'on peut constater l'effet poétique, expressif ou argumentatif du trope.
} 
du champ dérivationnel de "ciment" dans la presse Chypriote-grecque.

\subsection{Le Plan Annan : Traces énonciatives d'ironie et de dénonciation}

L'étude préalable du corpus révèle que lorsque les journalistes-scripteurs reprennent le verbe cimenter pour critiquer ou parler du plan Annan, le contenu de leur discours est ironique et désapprobateur. Par exemple, l'énoncé-titre d'un article paru le 18 janvier 2005 est particulièrement parlant :

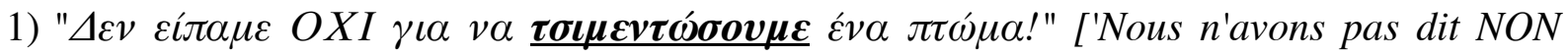 pour cimenter un cadavre!']}

Le journaliste se réfère explicitement à l'énoncé déclencheur. A y regarder de plus près, on s'aperçoit que cimenter dans cimenter un cadavre actualise la signification référentielle, ce qui implique la fonction cognitive de la métaphore. Le mot 'cadavre' utilisé métaphoriquement pour désigner ce plan, permet d'inhiber le sème /force/ actualisé par le verbe cimenter tel qu'il est utilisé dans l'énoncé déclencheur, en activant par propagation d'autres sèmes liés à la mort et donc à la négativité.

L'extrait suivant de l'article est révélateur de l'attitude dénonciatrice adoptée par le journaliste: ['Ces dirigeants refusent de comprendre la dynamique européenne ayant été développée après le NON salutaire au cadavre Annan, et ne respectent le verdict du peuple. Sans volonté,

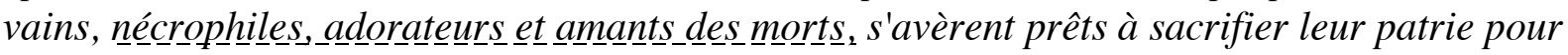
leur petitesse politique. Mais le peuple n'a pas dit NON pour cimenter un cadavre qu'il a déjà enterré. Ils doivent faire attention!'21] La présence de cette série d'images liées à la mort, donne lieu à une métaphore filée ("nécrophiles, adorateurs et amants des morts", "un cadavre qu'il a déjà enterré") et traduit l'intention du journaliste-scripteur qui consiste à souligner la non opérationnalité du plan onusien. Précisément, les propos suggèrent qu'il n'y ait plus rien à 'cimenter' dans le plan onusien, dont le rejet par la communauté chypriote grecque est définitif et irrévocable.

On le voit, l'énoncé déclencheur négativement reçu par la majorité de la communauté chypriote grecque confère au signifiant cimenter des connotations négatives et ironiques. L'énoncé-titre d'un autre article paru le 25 septembre 2005 renforce ce constat :

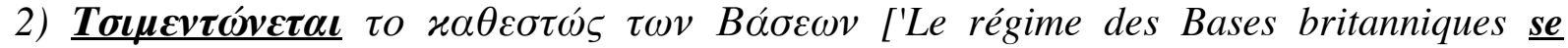 cimente']}

L'énoncé sous-titre qui le suit sert d'explicitation, et met en saillance l'attitude dévalorisante adoptée par le journaliste vis-à-vis du plan en question: ['L'approbation du plan Annan, promeut l'Accord de l'Instauration ${ }^{22}$ ]. Le premier paragraphe de l'article commence par la reprise du verbe cimenter dans son emploi métaphorique : ['Le régime des Bases britanniques se cimente au cas où le plan Annan se fera accepter'. ${ }^{23}$ ] La reprise du verbe cimenter traduit une volonté d'insistance de la part du journaliste qui s'attache à attirer l'attention du lecteur, lui faire partager son angoisse et l'inciter à adopter son point de vue. Grâce au jeu intertextuel, le verbe se trouve réinvesti de traits négatifs, ce qui renforce davantage la désapprobation et les effets ironiques.

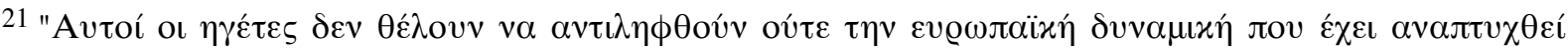

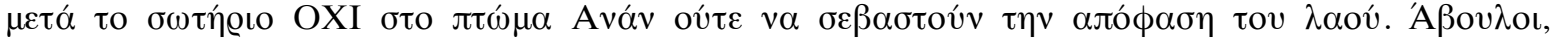

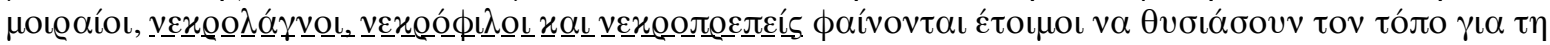

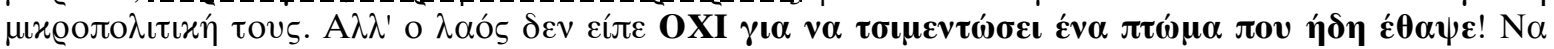

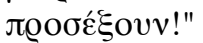

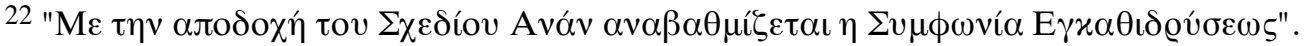

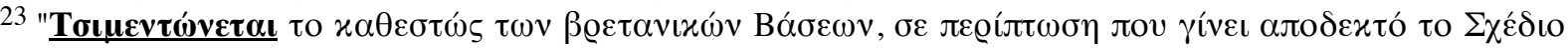
Avóv."
} 
Dans d'autres $\operatorname{cas}^{24}$, la métaphore est soutenue par d'autres verbes qui à première vue lui sont synonymes. Dans l'énoncé sous-titre d'un article paru le 19 avril 2004 on lit :

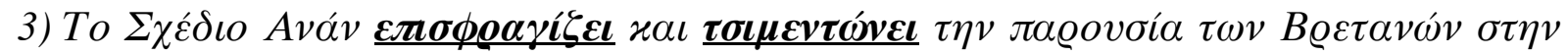
Kv́л@o ['Le plan Annan corrobore et cimente la présence des Britanniques à Chypre'.]

Les verbes $\varepsilon \pi \iota \sigma \phi \varrho \alpha \gamma i ́ \zeta \varepsilon \iota$ "il corrobore" et $\tau \sigma \iota \mu \varepsilon v \tau \omega \dot{v \varepsilon \iota ~ " i l ~ c i m e n t e " ~ s u r ~ l e ~ p l a n ~}$ syntagmatique sont sémantiquement apparentés. Cela n'implique pas une relation de synonymie parfaite mais celle d'une parasynonymie, vu que les deux verbes ne produisent pas ici le même sens. Ils s'entrecroisent et se condensent sémantiquement. La conjonction de coordination $x \alpha \iota$ "et" qui relie les deux verbes marque en effet la valeur additionnelle et complémentaire du verbe cimenter ; $\tau \sigma \iota \mu \varepsilon v \tau \omega \nu \varepsilon \iota$ "il cimente" densifie la fonction du premier verbe dont la signification lexicologique est connue par les lecteurs hellénophones. En outre, le premier verbe sert à éclairer contextuellement le sens du verbe cimenter par activation et inhibition sémiques. On observe une actualisation des sèmes /stabilité/, /force/ et /permanence/. Par ailleurs, le point de vue de l'énonciateur sert à inhiber les sèmes /positif/ et /mélioratif/ pour les remplacer par les sèmes /négatif/ et /dépréciatif/.

La présence du verbe cimenter ne sert pas seulement d'emphase expressive ou rhétorique, mais elle tend à amplifier la valeur argumentative du contenu de l'article.

Dans d'autres cas, le sens du verbe cimenter paraît plus vague, ce qui conduit à avancer plusieurs hypothèses interprétatives. Dans l'énoncé sous-titre qui suit le titre ['Omirou : des changements drastiques ${ }^{25}$ ] le journaliste-scripteur écrit :

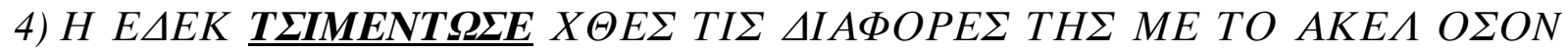
AФOPA TO $\Sigma X E \Delta I O$ ANAN (24 janvier 2005). ['L'EDEK ${ }^{26} \underline{\text { A CIMENTE HIER SES }}$ DIFFERENCES AVEC I'AKEL AU SUJET DU PLAN ANNAN"27]

Le verbe tel qu'il est utilisé ici semble s'éloigner largement de l'emploi fait par Christofias. Cimenter ne peut se substituer aux verbes renforcer ou consolider. Néanmoins, sans lire la suite de l'article, le lecteur peut associer l'emploi métaphorique à d'autres termes qui sur le plan paradigmatique lui sont sémantiquement apparentés, tels que confirmer, souligner ou déceler, faire connaître ou faire découvrir. Or, la lecture de la suite du texte fait basculer ce parcours interprétatif et oblige à chercher d'autres cheminements de lecture. Précisément, le journaliste poursuit en nous informant que : ['Le président du Mouvement (parti socialiste) Giannakis Omirou parlant hier [...] a confirmé une fois de plus la divergence des opinions entre son parti politique et l'AKEL, à l'égard de l'affaire de Chypre'. ${ }^{28}$ ] A y regarder de plus

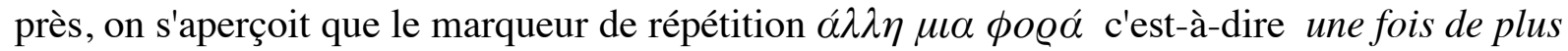
placé après le verbe $\varepsilon \pi \iota \beta \varepsilon \beta \alpha i \omega \sigma \varepsilon$ 'il a confirmé' annule ce parcours et complique davantage cette aventure d'interprétation. Certes, l'énoncé renvoie directement à l'énoncé déclencheur, qui peut servir ici d'ancrage interprétatif, mais renforcer recouvrirait-il la surface conceptuelle du signifiant "cimenter" dans le contexte précis? A notre avis, une telle hypothèse interprétative n'est pas solide, car la métaphore conceptuelle "cimenter c'est renforcer" ne

\footnotetext{
${ }^{24}$ Dans l'ensemble des occurrences inventoriées, nous avons croisé 7 cas où le verbe "cimenter" est soutenu sémantiquement par des synonymes contextuels; parmi les quasi-synonymes nous mentionnons les verbes $\varepsilon \mu \pi \varepsilon \delta \omega \nu \omega$ "consolider", "donner du sang".

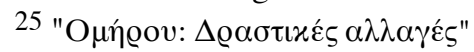

${ }^{26}$ EDEK est le sigle d'Eniea Dimokratiki Enosis Kyprou (ou Kentrou) (en anglais United Democratic Union of Centre). Il s'agit en effet du Mouvement pour la Démocratie Sociale dont le président actuel est Yannakis Omirou.

${ }^{27}$ Les majuscules sont du journaliste.

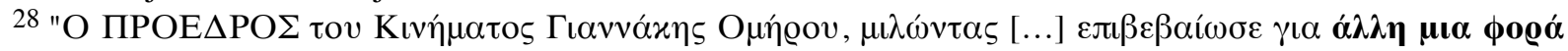

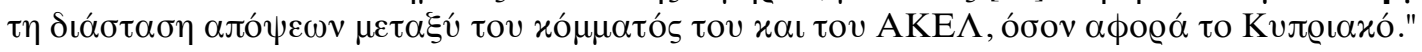


semble pas applicable dans ce cas. Nous serons donc amenée à chercher d'autres éléments de signification qui ne s'actualisent pas forcément dans le verbe renforcer. Dans cette optique, l'activation des sèmes /définitif/ ou /irrévocabilité/ semble mieux répondre aux conditions interprétatives imposées par le texte. Vu sous cet angle, le verbe cimenter s'identifie textuellement et sémantiquement aux verbes sceller ou cacheter auxquels le sème /irrévocabilité/ est inhérent. Bien qu'il y ait éloignement sémantique par rapport à l'énoncé déclencheur, le renvoi intertextuel n'est pas interdit. En revanche, le journaliste semble réinvestir et renforcer sémantiquement l'emploi métaphorique du verbe par rapport au co(n)texte initial.

\subsection{Syllepse : 'cimenter' l'occupation au propre et au figuré}

Les événements politiques, notamment après le rejet du plan Annan, se déroulent d'une rapidité fulgurante. L'un de ces événements est la 'cimentation' des terres, des propriétés de réfugiés chypriotes grecs. La communauté chypriote turque dans la tentative de se développer sur le plan économique s'approprie les terres appartenant aux Chypriotes grecs pour construire des bâtiments et des maisons de luxe. Exploitant cette réalité pesante et accablante pour la communauté chypriote grecque, les journalistes dans une volonté d'ironiser la situation font appel une fois de plus à la métaphore du ciment ${ }^{29}$ :

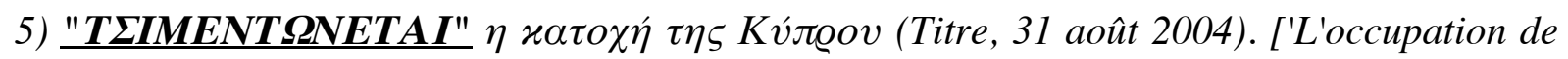
Chypre "se cimente"']

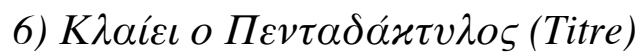

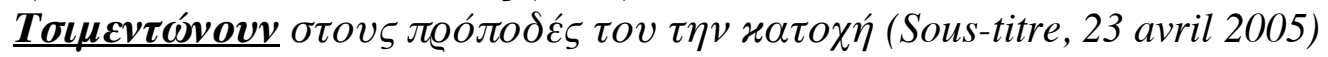

['Le Pentadactyle pleure'

'Aux pieds du Pentadactyle ils cimentent l'occupation'.]

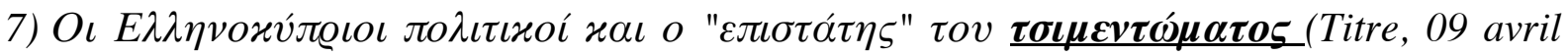
2006). ['Les hommes politiques chypriotes grecs et le "superviseur" de cimentation']

Dans (5), (6) et (7) les journalistes se réfèrent aux activités de construction qui se mobilisent dans la partie occupée. Dans (5), il s'agit de l'industrie du ciment appelée "Vasilikou", et comme le nom l'indique, il est question d'une industrie chypriote grecque. L'industrie du ciment chypriote grecque a pour but d'étendre ses activités dans la partie occupée. C'est ce qu'annonce d'ailleurs son directeur à Simerini. Le fait de fournir du ciment dans la partie occupée de l'île, durant une période où le développement des activités de construction et l'exploitation des propriétés chypriotes grecques dans la partie occupée atteignent leur point culminant, suscite inéluctablement des réactions.

Sans doute, dans (5) l'emploi du signifiant dans le contexte fait syllepse. Le trope de syllepse qui consiste dans l'utilisation d'un même mot, d'une même expression, tantôt dans son sens propre, tantôt dans son sens figuré, exerce ici une fonction argumentative. Les deux sens s'actualisent en même temps et l'un semble densifier l'autre. Ce sont les sèmes /désapprobation/ et /négativité/ qui s'activent, en inhibant par allusion le sème /mélioratif 30 / de l'énoncé déclencheur. Le jeu intertextuel est manifeste, ce qui renforce davantage les traces d'ironie. On observe également une mise entre guillemets du verbe. On peut postuler que l'emploi des guillemets révèle la co-présence du sens figuré et du sens propre et/ou sert à marquer la "mise à distance" (Authier-Revuz 1981: 127) du journaliste-scripteur.

\footnotetext{
${ }^{29}$ Dans l'ensemble de notre corpus, 16 occurrences semblent actualiser les deux sens.

${ }^{30}$ Cependant, il convient de noter que le trait /mélioratif/ ne s'actualise que du côté de l'instance émettrice et non pas dans l'ensemble des instances réceptrices.
} 
Précisément, dans (6) le sens propre intervient en premier, vu que le journaliste se réfère explicitement à un événement réel provoquant des réactions de la communauté chypriote grecque. L'absence de guillemets peut s'expliquer, à nos yeux, par le fait que la métaphore en question ait subit au fil du temps une sorte de 'fossilisation' ou de sédimentation dans le discours médiatique et politique chypriote grec. Le journaliste commence son article par les propos suivants : ['Sur le Pentadactyle, les Turcs cimentent littéralement l'occupation, grâce à la présence du drapeau du régime d'occupation, d'un côté, et à l'essor du bâtiment, de l'autre. La Chaîne de la radiophonie de Chypre a projeté des images tristes de destruction et de désacralisation de la vue de Pentadactyle, provoquées par les grands entrepreneurs qui tentent de résoudre le problème de Chypre en définitive et enraciner l'occupation à l'aide du ciment $^{\prime}{ }^{31}$ J La lecture attentive de ce paragraphe nous amène à observer que l'emploi répétitif n'est aucunement innocent. L'adverbe "littéralement" ou "à proprement parler" fonctionne comme actualisateur du sens propre, cependant ce qui suit interdit une lecture monolithique du seul sens "littéral". En effet, on constate l'actualisation d'une co-actualisation des deux significations qui se superposent et se densifient réciproquement.

Dans (7) la critique virulente exercée par le journaliste vise certains hommes politiques Chypriotes grecs. Alors que les hommes politiques s'opposent à la 'cimentation' des biens des Chypriotes grecs dans la partie occupée, leurs actes contredisent leur discours. Le journalisteénonciateur poursuit en ces termes: ['Un jour ils se divertissent au sein des concerts coorganisés par les deux communautés avec le consentement du régime d'occupation [...] l'autre jour délibèrent avec le gouvernement turc, et puis font la fête avec Talat, le "superviseur" de la cimentation organisée dans la partie occupée. Mais pourquoi ne se mettent-ils pas en colère contre le 'superviseur' d'Ankara dans la partie occupée, qui surveille la cimentation des chypriotes grecs et en la favorisant? $\left.{ }^{132}\right]$

On remarque que la cimentation organisée consiste à défiger le syntagme nominal figé crime organisé : la substitution lexicale est sans doute délibérée et renvoie implicitement à crime organisé. Cela réinvestit l'emploi métaphorique de "cimentation" de traits péjoratifs.

\subsubsection{Défigement et ironie}

Une autre stratégie mise en œuvre par les journalistes est le défigement ${ }^{33}$. Ce procédé qui se ramène à un jeu de mots peut être soit linguistique soit culturel. Quant au premier type, les locutions, les mots-composés, les proverbes etc. sont des figements ou des "jeux de mots basés sur ces figements, des défigements" (Sullet-Nylander 1998 : 204). Chez G. Gross (1996 : 20), "le figement peut être mis en évidence grâce à l'effet provoqué par le jeu du défigement, qui consiste à briser le carcan qui caractérise les suites figées." Un défigement culturel $^{34}$ tient à l'évocation des instances culturelles qui s'impriment dans et par des expressions figées.

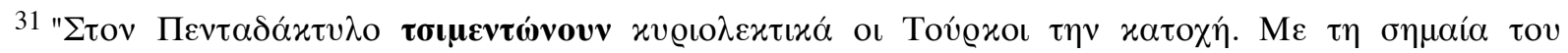

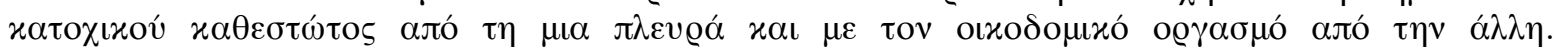

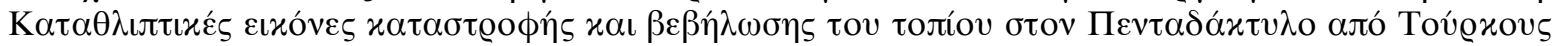

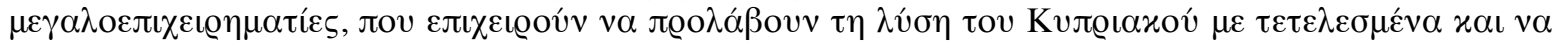

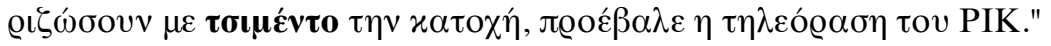

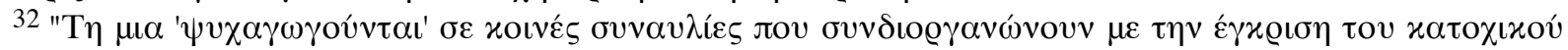

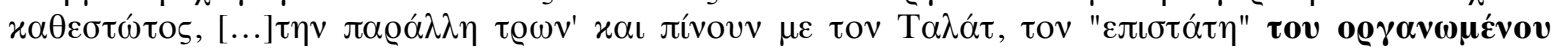

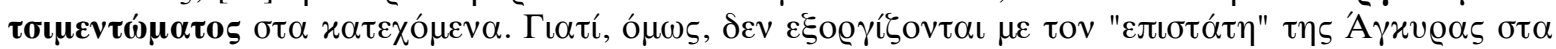

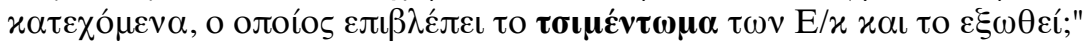

${ }^{33}$ Nous n'avons recensé que deux cas de défigement, ceux que nous présentons ici.

34 'En attendant Godot' est à l'évidence un figement culturel et 'En attendant Godart' en est le défigement (exemple emprunté à Sullet-Nylander 1998: 209f.).
} 
du champ dérivationnel de "ciment" dans la presse Chypriote-grecque.

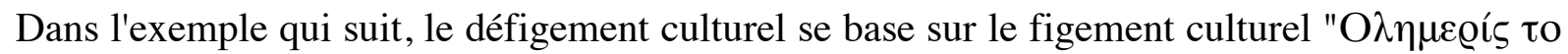

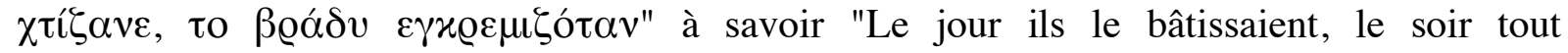
s'écroulait":

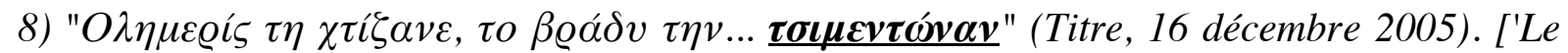
jour ils la bâtissaient, le soir ils le... cimentaient']

Le choix de ce défigement n'est pas en soi aléatoire. Au contraire, c'est par ce jeu de mots que le journaliste évoque l'expression d'origine, ce qui favorise une superposition des parcours interprétatifs. Précisément, l'énonciateur fait allusion au pont d'Arta dont la légende est connue de tous les Grecs. Le Pont d'Arta est une ancienne ballade populaire qui raconte la naissance difficile du pont d'Arta. Les recherches démontrent que la légende cache en elle une réalité historique. Lorsqu'il devient nécessaire pour une grande partie de l'armée turque de passer par la région d'Arta, les habitants seront convoqués pour bâtir un pont. Bien des Grecs se précipitent pour manifester leur volonté dans le but de gagner une faveur. Mais, lorsque le vrai objectif des Turcs se décèle, les habitants y vont toutes les nuits pour détruire ce qu'ils bâtissent tout au long de la journée.

Au-delà de l'emploi métaphorique du ciment qui contribue à l'ironie, nous constatons que ce défigement culturel vient, par les démultiplications sémantiques et l'implicite culturel qu'il actualise, renforcer davantage les effets de sens ironiques. Ici s'exerce une allusion au passé, à l'histoire touchant aux relations entre Grecs et Turcs, qui perdure depuis des siècles. Néanmoins, nous observons que la situation se renverse; cette fois-ci ce sont les Turcs qui bâtissent et non pas les Grecs. L'emploi du temps considéré comme faisant appel à la mémoire collective, s'avère tout de même crucial.

Notre analyse met en saillance une stratégie discursive qui favorise un effet paradoxal. Le journaliste-scripteur vise à ironiser le projet des Turcs dans la partie occupée. Le but des Turcs dans la rue Ledra, écrit-il, se dévoile au fur et à mesure. La lecture de la suite de l'article corrobore ce parcours interprétatif : ['Les vrais objectifs de la partie turque dans la rue Ledra se décèlent au quotidien. Le côté turc insiste avec obstination orgueilleuse sur le pont qu'il est en train de fabriquer et figer à tout prix, en dépit des réactions des Chypriotes turcs mêmes. Aussitôt les jours passent ... il le cimente de plus en plus, tout en asphaltant la route autour du pont, pour assurer le passage des véhicules de l'Attila turc'.35] Aux yeux de l'énonciateur le pont construit dans la rue Ledra est devenu le nouvel emblème de l'occupation turque. Comme on le voit, le journaliste, dans le paragraphe qui suit le titre, met en place la métaphore du ciment, tout en employant des verbes qui lui sont contextuellement synonymes ( $\pi \alpha \gamma \iota \omega ́ v \varepsilon \iota$ 'il fige' et $\alpha \sigma \phi \alpha \lambda \tau o \sigma \tau \varrho \omega ́ v \varepsilon \iota$ 'il asphalte'). Un examen attentif de l'axe syntagmatique permet de voir l'actualisation des traits /dépréciatif/, /négatif/, /permanence/ et /irrévocabilité/ dans les trois verbes. Dans ce jeu de trocs et de condensation sémantiques les signifiants témoignent d'une critique virulente.

H. Svensson (2004 : 121) observe que "Pour qu'un défigement soit réussi, il faut donc que l'expression d'origine soit évoquée, même si ce n'est que par association." En effet, le jeu de mots qui repose sur le processus de défigement rappelle le figement culturel sur lequel il se base, ce qui active les effets ludiques ${ }^{36}$. En ce sens, le défigement culturel déjà étudié ainsi que le défigement linguistique qui suit sont jugés réussis.

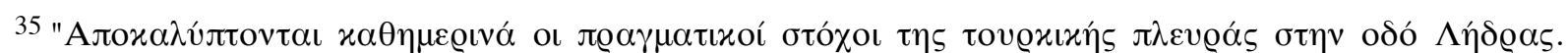

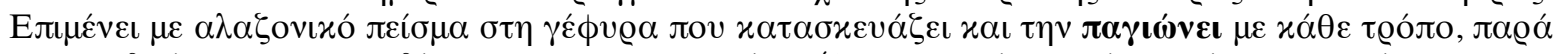

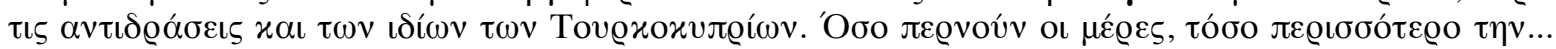

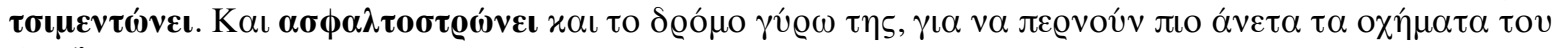

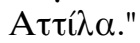

36 Rastier fait remarquer (1997 : 309-310) : "l'on n'a le choix qu'entre figement et défigement, c'est sans doute par le défigement qu'un locuteur peut au mieux manifester sa liberté. Dans la mesure où le lexique est de la
} 
Ainsi :

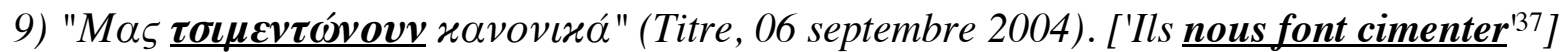

Il suffit d'une simple observation du titre, pour y voir l'amorce de l'idée qui met en débat un paradoxe se produisant au sein de la communauté chypriote grecque. L'énonciateur se réfère explicitement aux activités de l'industrie chypriote grecque "Vasilikou" (voir l'exemple 5).

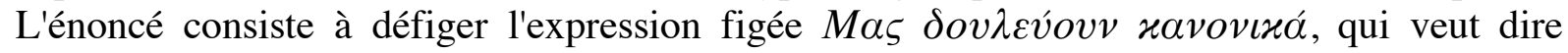
grosso modo "ils nous font marcher". Il s'agit d'une syllepse vu qu'il correspond en même temps à l'actualisation du sens propre et du sens figuré du verbe. La syllepse fonctionne ici comme trope. Elle entretient dans le texte le rire et l'ironie, en mettant en saillance l'attitude dénonciatrice du journaliste vis-à-vis du comportement de certains Chypriotes grecs. Il convient de souligner que le "nous" étoffé par le présent fonctionne comme un embrayeur du discours, susceptible d'impliquer l'expérience commune, ainsi que la mémoire collective. De même, ce pronom personnel peut être le corréla instantané d'un "vous" implicite, qui vise à mettre en éveil le public visé, en l'occurrence toute la communauté chypriote grecque.

La suite de l'article dévoile que le recours au verbe cimenter est incident sur l'opinion personnelle du journaliste qui s'efforce de dépeindre la réalité crue : ['Pour mieux cimenter l'illégitimité provocante, la liquidation de notre terre, l'exploitation illégale de la terre hellénique'. $\left.{ }^{38}\right]$ Le principe de répétition devient à son tour un corollaire du principe d'insistance. Dans le paragraphe qui sert de péroraison, le journaliste emploie ce verbe deux fois. La première récurrence actualise le sens littéral alors que la deuxième le sens métaphorique : ['Il paraît que certains ne se sont pas décidés qu'à mieux cimenter avec leur aide nos propriétés dans la partie occupée mais se sont déterminés à cimenter l'affaire à travers leur silence' ${ }^{39}$ ]

\subsubsection{Cimenter la non solution}

Dans notre perspective d'analyse intertextuelle, il nous revient de noter que les messages que les journalistes-scripteurs visent à transmettre, s'enchaînent et se renforcent mutuellement. Cimenter la non-solution s'oppose au contenu de l'énoncé Cimenter le Oui. Ainsi dans un article dont l'un des intertitres est intitulé :

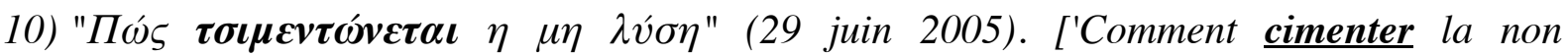
solution ?'], l'emploi métaphorique du verbe cimenter fait inéluctablement écho à l'énoncé déclencheur. Il parle de la 'cimentation' de la non-solution. Les verbes garantir, assurer ou consolider semblent être plus proches sémantiquement du verbe cimenter ici. En fait, tout l'article parle du renforcement de la situation économique des Chypriotes turcs qui creuse davantage la séparation des deux communautés. Le journaliste finit par réutiliser le verbe dans la phrase ['Bref, le renforcement de la situation économique des Chypriotes turcs cimente la non solution et la séparation. Pour dire la vérité crue : Nous creusons notre

doxa figée, le défigement des locutions aura un effet quelque peu subversif. Comme le paradoxe, il paraît contester les normes qui ont présidé au figement. Ou encore, et corrélativement, il a un effet ludique, et passe pour un jeu de mots."

$37 \mathrm{Au}$ lieu de rendre l'énoncé en question par une traduction littérale ('ils nous cimentent complètement'), nous avons jugé nécessaire de faire appel à une traduction pouvant transmettre l'implicite et les connotations moqueuses actualisées par l'énoncé. D'où notre prédilection pour la traduction "ils nous font cimenter", dans le but d'évoquer l'expression française "faire marcher quelqu'un". Une autre solution basée sur le figement "mener quelqu'un en bateau" serait "ils nous cimentent en bateau".

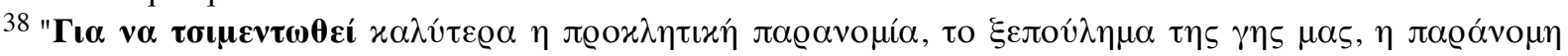

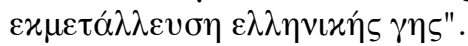

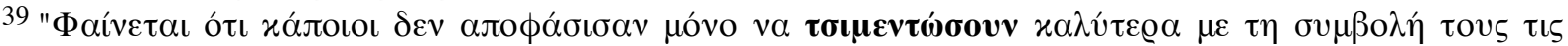

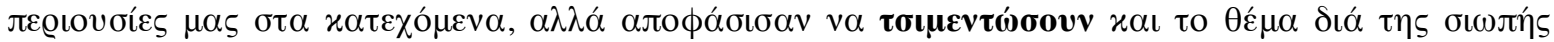
tous". 
du champ dérivationnel de "ciment" dans la presse Chypriote-grecque.

tombeau'.40] Cela revient à admettre que les propos du journaliste témoignent d'une mise en garde et d'un regard méfiant envers la situation actuelle. L'emploi de la métaphore vient actualiser des traits négatifs, tout en reflétant une prise de position aussi bien politique qu'idéologique affichée du scripteur.

On l'a déjà vu, le point de vue de l'énonciateur est susceptible d'inverser les valeurs. Dans l'exemple qui suit les sèmes actualisés ne sont que ceux relevant de la négativité, de l'irrévocabilité, et de la désapprobation. ['Les camions et les voitures privées chypriotes turcs, inscrits dans le régime illégal de l'occupation sont autorisés à circuler librement dans les régions libres. Des milliers des Chypriotes grecs donnent du sang à et cimentent grâce à leurs millions leur conquérant et abatteur. Et cela ne semble déranger personne. Ni lois ni état n'existent. Quant à la dignité et au patriotisme, ceux-ci s'enterrent sous un chypriotisme honteux, portant le sceau des efforts ridicules de rapprochement de l'AKEL, avec la synergie du DISY, un parti autrefois patriotique'.41]

L'énonciateur s'efforce de persuader son public en montrant que ce qui est à dénoncer c'est l'attitude irréfléchie et imprudente adoptée par certains Chypriotes grecs. Les choix lexicaux et syntaxiques (par ex. "régime illégal", "donnent du sang", "leur conquérant et abatteur") mettent en évidence les intentions du scripteur. Le verbe néologique $\alpha \iota \mu o \delta o \tau \omega$, (dérivé de $\alpha i ́ \mu \alpha$ "sang" et du verbe $\delta i ́ \delta \omega$ "donner") qui précède celui de cimenter et que nous traduisons littéralement par "donner du sang" acquiert dans le contexte un sens métaphorique. Le verbe en question sert de "stabilisateur" de sens. Il comporte comme le verbe cimenter le sème /force/ ou /renforcement/, ce qui entraîne une isotopie. Il revient de dire que les éléments linguistiques qui entourent les deux verbes ('leur abatteur', 'chypriotisme honteux' annulent le sème /mélioratif/ tout en actualisant les traits /dépréciatif/ et /négatif/). Les valeurs de 'dignité' et de 'patriotisme' semblent aux yeux du journaliste être enterrés et remplacés par un 'chypriotisme honteux'. L'expression 'chypriotisme honteux' est en soi burlesque et implique la prise de position du scripteur.

Tout au long de l'article, on constate que le journaliste se réfère directement aux tentatives de réunification de la part de l'AKEL, ce qui fait émerger l'emploi intentionnel du verbe cimenter. Par ce jeu intertextuel, le journaliste vise à caricaturer et ridiculiser les efforts faits par le parti politique de gauche (AKEL) ainsi que par le parti politique de droite (DISY). Par là-même, la métaphore ad hoc réinvestie des connotations ironiques et moqueuses permet au journaliste de disqualifier les Chypriotes grecs qui se rendent très souvent à la partie occupée, pour se promener, visiter les casinos chypriotes turcs, et y laisser leur argent.

\subsection{La métaphore du ciment et réalités mises en mot}

La métaphore du ciment dans le même journal sert également à décrire ou mieux encore à créer une autre réalité. Il s'agit donc pour nous de cerner d'autres énoncés tirés des articles ne touchant pas au plan Annan, mais à d'autres situations socio-politiques.

La métaphore dans son emploi désormais généralisé peut être considérée comme étant un réservoir de mise en actes et d'implicites, qui semblent servir le discours médiatique. Aussi parlera-t-on d'une métaphore reposant sur la mémoire collective qui prédétermine le discours

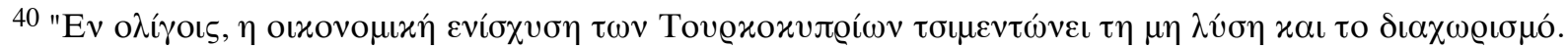

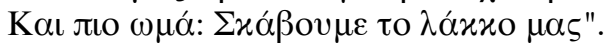

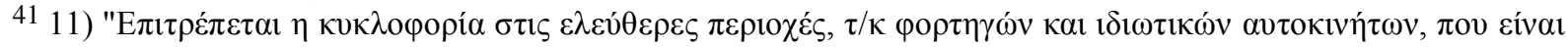

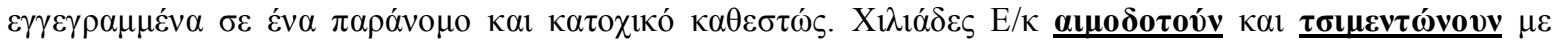

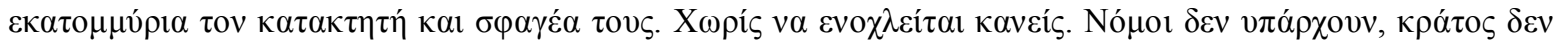

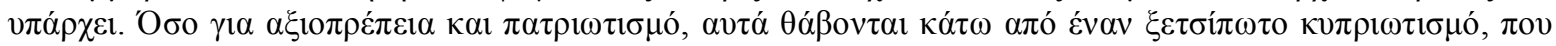

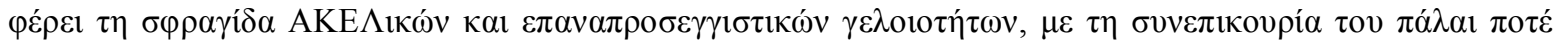

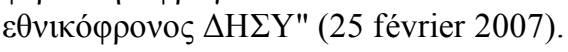


et conditionne ou préjuge le regard que l'on porte sur les faits. Nous pouvons également avancer l'idée que dans les énoncés qui se rapportent à d'autres cas que le plan Annan et le problème politique de Chypre, la métaphore du ciment paraît parfois plus opaque ou fonctionne comme un oscillateur sémantique répondant à des "sens situationnels".

L'énoncé sous-titre d'un article paru le 18 juillet 2004 est particulièrement éloquent ${ }^{42}$ :

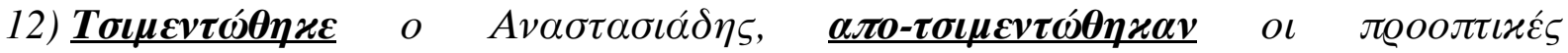
['Anastasiadis s'est cimenté, les perspectives se sont dé-cimentées']

Le journaliste recourt à une dérivation ${ }^{43}$. En rhétorique, cette stratégie discursive désigne le processus qui consiste "à utiliser dans une même phrase des mots, dérivés d'un même radical" (Ricalens-Pourchot 2003 : 154). Autrement dit, elle vise à rapprocher dans le discours des mots appartenant à la même famille. (cf. Bacry 1992 : 196). Il s'agit d'un emploi délibéré, dans le but de créer des effets de sens, et de marteler une idée. Reposant sur ce jeu de captation, l'énonciateur-dénonciateur se propose de nommer et/ou d'interpréter autrement une "réalité" politique qui consiste selon lui dans la "cimentation" du président du parti politique DISY et la "dé-cimentation" des possibilités pour ce parti d'y revenir et revendiquer la présidence. C'est d'ailleurs ce que dévoilent ses propos méticuleusement et sciemment élaborés : ['Le grand problème du DISY réside dans le fait qu'il a dé-cimenté toutes possibilités d'y revenir et revendiquer la présidence'.]

Il va de soi que la métaphore du ciment s'avère plus que jamais constitutive de la "réalité politique". Elle assume une fonction d'amplification qui vise à maximiser l'intentionnalité du scripteur. Par le processus de dérivation, un jeu de mots se produit, ce qui favorise des effets ludiques et ironiques. Cet emploi, fonctionnant comme marqueur d'adhésion met en évidence la posture de l'énonciateur, qui semble s'opposer à ce qui se passe au sein du parti politique DISY. L'affixe /apo/ qui équivaut à son homologue français /dé/ défige le sens initial du verbe "cimenter", tout en renforçant les connotations péjoratives, négatives et ironiques de l'article. On observe une actualisation des sèmes /négativité/, /péjoration/ et /destruction/.

Son emploi traduit une volonté d'emphase et d'accentuation, ce qui favorise une hyperbole. Le signifiant néologique /dé-cimenter/ peut s'expliquer par le biais d'autres verbes tels que détruire ou dissiper.

Comme on le voit, la métaphore du ciment présente une opacité sémantique ; cependant le recours au contexte est sans équivoque salutaire.

Ainsi :

13) $\Sigma \tau \eta v ~ E \lambda \lambda \alpha ́ \delta \alpha, \pi \alpha \varrho \alpha \iota \sigma o ́ v \tau \alpha \iota . ~ \Sigma \tau \eta v ~ K v ́ \pi \varrho o, ~ \boldsymbol{\tau \sigma \iota \mu \varepsilon v \tau \omega ́ v o v \tau \alpha \iota ! ~ ( T i t r e , ~} 24$ septembre 2004). ['En Grèce ils démissionnent. A Chypre ils se cimentent!']

Dans l'énoncé-titre ce sont les rapports syntagmatiques qui servent à stabiliser le sens. Le verbe $\pi \alpha \varrho \alpha \iota \tau o v ́ v \tau \alpha \iota$ c'est-à-dire "ils démissionnent" fonctionne comme l'antonyme ${ }^{44} \mathrm{du}$

42 Dans l'ensemble du corpus, nous avons retrouvé le même jeu de dérivation, dans un autre contexte, qui ne concerne pas l'affaire chypriote mais la vie politique interne. L'allusion à l'énoncé déclencheur est évidente, vu que le journaliste se réfère à $\mathrm{D}$. Christofias de manière ironique : Cette fois-ci, Dimitris Christofias a réussi à faire entendre une seule voix au sein des médias de la Gauche : Il cimente le métropolite de Kykkou pour dé-

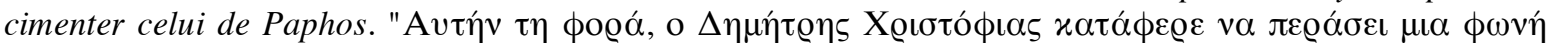

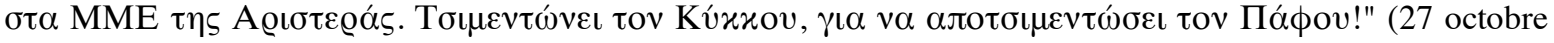
2005)

${ }^{43}$ En grammaire traditionnelle, la dérivation est "un procédé de formation de mots nouveaux (morphologie) par ajout d'affixes à un mot appelé base" (Rey-Debove et al. 2003: 702).

${ }^{44}$ En ce qui concerne l'antonymie, nous avons repéré un autre cas où le verbe affaiblir vient désambiguïser le

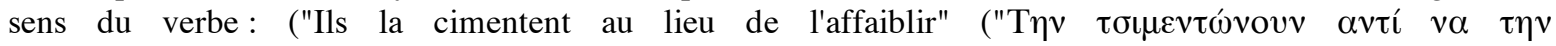

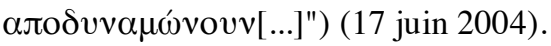


du champ dérivationnel de "ciment" dans la presse Chypriote-grecque.

verbe cimenter et sert à désambiguïser le sens du verbe en question. Le journaliste veut insister sur le chaos dominant la vie politique de Chypre. En poursuivant, l'énonciateur souligne: ['Dans la république bananière chypriote, l'insensibilité politique, fruit d'inexistence de santé politique et de comportements dépourvus de responsabilité se cimente comme vertu'. $\left.{ }^{45}\right]$

Tout l'énoncé mentionné ci-dessus contribue à la concrétisation du contenu que le journaliste veut conférer au verbe en question. Le journaliste-ironiste adopte une attitude dévalorisante voire sarcastique, en invitant le lecteur à poursuivre le même chemin. On observe un renversement de valeurs: "comme vertu politique" s'oppose au "régime bananier", à "l'insensibilité politique". Le point de vue de l'énonciateur est décisif. Le sens du verbe, notamment dans la dernière récurrence semble flou et opaque. A y regarder de plus près, on constate que plusieurs sèmes s'actualisent: il y a actualisation des sèmes de /force/, de /consolidation/, et de /régime/ et rupture avec le trait /positif/ de l'énoncé déclencheur. Il convient de souligner que le verbe cimenter mis au passif présente ici un cas particulier qui rappelle dans son usage textuel et sémantique l'exemple 12 .

La métaphore du ciment s'emploie également dans des contextes qui ne s'associent ni au plan onusien ni au problème politique de Chypre ${ }^{46}$. Voici quelques exemples :

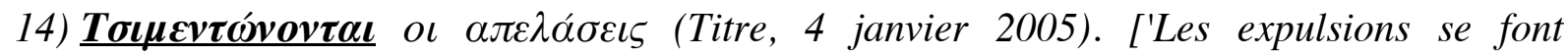 cimenter']}

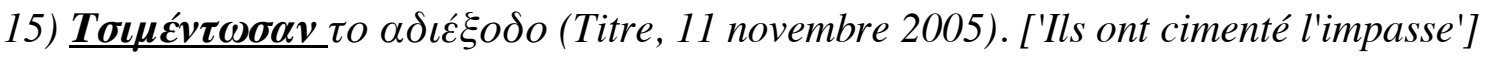

Le premier cas fait émerger un autre emploi qui s'éloigne des exemples précédents. Ici le journaliste se rapporte au cadre juridique qui a été adopté par le pouvoir judiciaire au sujet des expulsions. On observe une actualisation des traits positifs; l'énoncé ne témoigne d'aucune attitude dévalorisante. Il s'agit pour le journaliste de nous informer que la législation et les dispositions judiciaires à l'égard des expulsions des étrangers qui ne semblent pas se conformer à la loi chypriote deviennent plus restrictives. A l'évidence, le renvoi à l'énoncé déclencheur est contredit par le contexte. Il semble assumer une fonction expressive, créée par la fréquente utilisation du verbe en question.

Dans l'exemple 15, il n'en est pas ainsi. L'impasse entre les producteurs de pommes de terre et le gouvernement doit désormais être considérée comme "cimentée", vu que les deux parties ne semblent pas s'en aviser. Les traits qui s'actualisent sont ceux de /négativité/ et de /stagnation/.

Dans d'autres cas, les journalistes reprennent le verbe en mettant ensemble le NON.

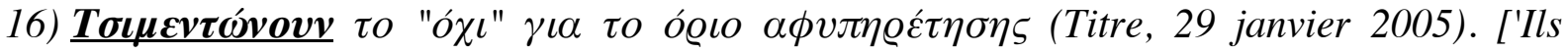
cimentent le "non" pour le taux de retraite']

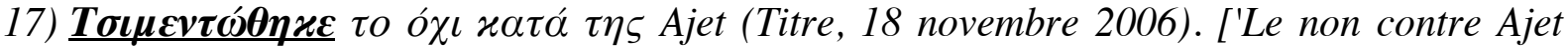
s'est fait cimenter']

En dépit de la rupture constatée sur le plan pragmatique des deux énoncés ci-dessus avec l'énoncé déclencheur, le renvoi implicite à l'énoncé déclencheur n'est point interdit. Dans le premier cas, on remarque une mise entre guillemets du non, ce qui traduit soit un emploi flou soit un emploi délibéré. Au non est conféré un sens métonymique, puisqu'il connote le refus, l'attitude dénonciatrice des enseignants vis-à-vis de la décision du gouvernement d'augmenter

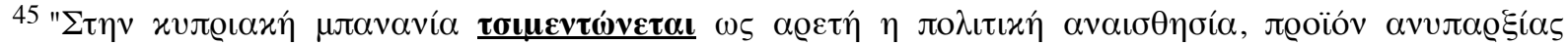

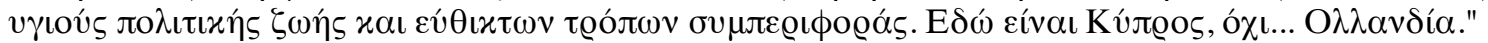

46 Dans notre corpus nous avons inventorié 16 occurrences dans des contextes ayant trait à d'autres situations socio-politiques qui ne sont liées ni à l'affaire chypriote ni aux relations entre partis politiques.
} 
le taux de retraite. En ce sens l'emploi métaphorique du verbe cimenter implique la résistance ainsi que le point de vue de l'énonciateur. Le deuxième cas porte sur le crash aérien qui s'est produit en août 2005 avec une compagnie aérienne chypriote grecque. A notre avis, l'emploi de ce verbe n'est point aléatoire; il implique au contraire une critique et une attitude dénonciatrice vis-à-vis de ce qui s'est produit à l'époque et de ce qui se passe aujourd'hui. On observe donc une prise de position implicite qui se voit à travers cette métaphore négativement marquée.

\subsection{En guise de conclusion}

Les pages qui précèdent mettent en évidence les jeux et enjeux textuels et intertextuels que la métaphore du ciment entraîne au sein d'un même journal. Elles démontrent que la métaphore étudiée se place en dehors du fait proprement linguistique. La métaphore s'appuie sur une relation intertextuelle et une donnée de l'expérience commune. Les moments intertextuels ne sont pas ponctuels, mais constitutifs. Il y a un enchâssement et un enchevêtrement de ces voix multiples qui semblent dialoguer entre elles et avec le lecteur. Cette métaphore semble se comporter comme une activité énonciative particulière qui procède par rapprochement des sèmes, des voix et des discours. Notamment dans sa mise en titre et sous-titre, elle devient véhicule de l'ethos, de la prise de position et du point de vue du journaliste-scripteur vis-à-vis des événements qui semblent lui tenir à coeur. Elle témoigne d'une remise en question, d'une méfiance et imprime l'opposition, la dénonciation, tout en mettant en saillance les crispations socio-politiques et idéologiques. Sous ce prisme, l'image du ciment constitue un outil d'interaction doté d'une fonction d'abord persuasive et évaluative. Les journalistes, en mettant en place la métaphore en question, désirent porter la "plume dans la plaie" de la communauté chypriote grecque. Sous cet angle, la métaphore n'est pas dénuée d'aspects manipulatoires, car elle a pour vocation d'agir sur l'auditoire et de l'inciter à adopter les points de vue des journalistes.

On peut donc déduire que l'usage circulaire de la métaphore fondé sur la réminiscence du passé récent est une pragmatique discursive qui "dirige" le lecteur. La métaphore du ciment semble bien servir le réel en le décrivant. On peut avancer l'idée qu'il s'agit, d'une part, des innovations sémantiques mises en place pour donner lieu à des 'nouvelles réalités' et d'autre part qu'il est question des stratégies destinées à répondre aux besoins rhétoriques et expressifs créés par ces nouvelles réalités. Vue sous cet angle, la métaphore du ciment vient remplir des "vides" sémantiques ou contextuels 'creusés' par l'énoncé déclencheur en question. L'énoncé initial apparaît donc comme le générateur des discours et le 'déclencheur' des besoins expressifs et argumentatifs.

Du point de vue du sens, l'étude met en saillance une métaphore productive, conduisant à une prolifération de sens ou peut-être de non-sens féconde. Cette métaphore dont le sens est loin d'être immuable, pose le problème de l'instabilité du sens. L'ambiguïté et l'opacité sémantique sont les plus grands problèmes qui se posent lors de son étude ; alors que la fonction de "cimenter" dans l'énoncé déclencheur semble être celle de la transparence sémantique, dans la majorité des autres énoncés elle semble floue, opaque et incertaine. Cela favorise les aléas de la communication et l'ambivalence des stratégies interprétatives mises en œuvre par le lecteur et l'analyste.

Mesurer les propriétés sémantiques supplémentaires que la métaphore acquiert au sein des articles étudiés conditionne donc une lecture réfléchie et méticuleuse pouvant prendre en compte tout facteur générateur du sens. Le recours au contexte est salutaire ; cela revient à dire que la métaphore du ciment ne peut se comprendre que dans son usage discursif et énonciatif aux prises avec le jeu intertextuel qui semble en principe la constituer. 
La complexité de cette métaphore ad hoc qui est historiquement et idéologiquement constituée ne favorise pas des généralisations théoriques. L'étude vient cependant confirmer la puissance argumentative de la métaphore, son potentiel sémantique à valeur transformatrice et redynamisante. Elle démontre l'importance du contexte pour l'interprétation de la métaphore qui est toujours susceptible de se recycler et se ranimer discursivement.

\section{Références Bibliographiques}

Authier-Revuz, Jacqueline (1981) : "Paroles tenues à distances". In: Conein, Bernard et al. (eds.) (1981): Matérialités discursives. Lille : 127-142.

Bacry, Patrick (1992): Les figures de style. Paris.

Barthes, Roland (1998/1972) : "La théorie du texte". In : Encyclopédie Universalis. Edition électronique, version 4. Paris.

Beardsley, Monroe (1962): "Metaphorical Twist". Philosophy and Phenomenological Research 22/3: 293-307.

Bourdieu, Pierre (1996) : Sur la télévision. Paris.

Collombat, Isabelle (2005) : Le discours image en vulgarisation scientifique. Etude comparée $d u$ français et de l'anglais. Thèse de Doctorat de Linguistique, Université de Laval.

Détrie, Catherine (2001) : Du sens dans le processus métaphorique. Paris.

Eco, Umberto (1973) : "Sémantique de la métaphore". Tel-Quel 55, 25-46.

Encyclopédia Universalis (éd.) (1998): Edition électronique version 4. Paris.

Furet, Claude (1995) : Le titre. Pour donner envie de lire. Paris.

Grize, Jean Blaise (1990) : Logique et langage. Paris.

Gross, Gaston (1996) : Les expressions figées en français. Noms composés et autres locutions. Paris.

Horne, Christine (1992) : "La métaphore. Quelques définitions contemporaines". ALFA 5: 179-190.

Jamet, Denis (2003) : "A rose is a rose is (not) a rose. De l'identification métaphorique?". Cycnos 21/1.

Kerzazi-Lasri, Rafika (2003) : La métaphore dans le commentaire politique. Paris.

Kocourek, Rostislav (1991) : La langue française de la technique et de la science, vers une linguistique de la langue savante. Wiesbaden.

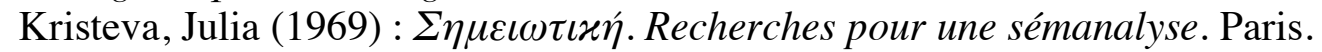

Landheer, Ronald (2001/2002) : "La métaphore, une question de vie ou de mort ?". SEMEN 15: Figures du discours et ambiguïtés : 25-39.

Murat, Michel (1981) : "La métaphore verbale. Une mise au point". Travaux de linguistique et de littérature 1: 327-346.

Ricoeur, Paul (1975) : La métaphore vive. Paris.

Ricalens-Pourchot, Nicole (2003) : Dictionnaire des figures de style. Paris.

Rastier, François (1987) : Sémantique interprétative. Paris.

Rastier, François (1989) : Sens et Textualité. Paris.

Rastier, François (1997) : "Défigements sémantiques en contexte". In: Martins-Baltar Michel (ed.) : La locution, entre langues et usages. Paris: 305-329. (= Signes).

Rey-Debove, Josette et al. (2003) : Le nouveau Petit Robert. Dictionnaire alphabétique et analogique de la langue française. Nouv. éd. mise à jour et augmentée. Paris: 702. (= Le nouveau Petit Robert 1).

Riffaterre, Michaël (1980) : "La trace de l'intertexte". La pensée 215: 4-18.

Sullet-Nylander, François (1998): Le titre de presse. Analyses syntaxique, pragmatique et rhétorique. Stockholm. (= Forskningsrapporter 8 ).

Swadesh, Morris (1955) : "Towards greater accuracy in lexicostatistics dating". International Journal of American Linguistics 21: 121-137. 
Svensson, Maria Helena (2004) : Critères de figement. L'identification des expressions figées en français contemporain. Thèse de doctorat, Umeå Universitet. (= Skrifter från moderna språk 15. Institutionen för moderna språk, Umeå Universitet). www.divaportal.org/diva/getDocument?urn_nbn_se_umu_diva-335-1_fulltext.pdf.

Todorov, Tzvetan (1981) : Mikhaïl Bakhtine. Le principe dialogique. Paris. 